\title{
Autoconcepto físico y estilos de vida en la adolescencia
}

\author{
Physical self-concept and lifestyles \\ in adolescence
}

Arántzazu RODRÍGUEZ*

Alfredo GOÑ I*

Sonia RUIZ DE AZÚA*

Fecha de Recepción: 15-06-2004

Fecha de Aceptación: 13-02-2006

\section{RESUMEN}

Los hábitos de vida saludables guardan relación con el autoconcepto fisico y también con el bienestar psicológico, al menos durante los años de la adolescencia. Esto es lo que confirman los datos que se presentan en este estudio, cuya muestra la componen 539 estudiantes de entre 12 y 23 años. Los participantes cumplimentaron el Cuestionario de Autoconcepto Físico (CAF), un cuestionario sobre conductas saludables de los escolares y el Cuestionario de Bienestar Psicológico (EBP). Los resultados sugieren diversos flancos por donde orientar una intervención psicosocial que ayude a promover el desarrollo personal y la convivencia social, desde una triple conexión: el estilo de vida, el bienestar psicológico y el autoconcepto fisico.

\section{PALABRAS CLAVE}

Autoconcepto fisico, Bienestar psicológico, Estilos de vida, Adolescencia.

\section{ABSTRACT}

Healthy lifestyles are associated with both physical self-concept and psychological well-being, at least during the adolescence. In this study the results confirm the relation.

\footnotetext{
* Facultad de Psicología. Universidad del País Vasco.
} 
Autoconcepto fisico y estilos de vida en la adolescencia

The participants were 539 students between 12 and 23 years old. The instruments used were the Physical Self-Concept Questionnaire (CAF), a questionnaire about healthy behaviors and the Psychological Well-being Questionnaire (EBP). The results suggest different ways of orienting a psychosocial intervention in order to get both personal development and social life, from a triple connection of lifestyle, psychological well-being and physical self-concept.

\section{KEY WORDS}

Physical self-concept, Psychological well-being, Lifestyle, Adolescence. 


\section{INTRODUCCION}

En esta sociedad nuestra, a la que se denomina con razón la sociedad de los individuos, logros como el estar en paz consigo mismo, la armonía interior, el bienestar psicológico o un autoconcepto positivo son muy apreciados; se trata de objetivos cargados de valor cuya consecución suele justificar esfuerzos y sacrificios (Goñi, 2000).

Pero, de otro lado y simultáneamente, vivimos en una cultura hedonista que transmite mensajes a favor de la satisfacción inmediata de los impulsos y apetencias. Muy en especial durante el periodo de la adolescencia, las personas se enfrentan de continuo a nuevas situaciones que les ofrecen la posibilidad de consumir bebidas alcohólicas, de fumar o de adoptar otras muchas conductas de riesgo. La adolescencia es una etapa de la vida en la que hay que ir decidiendo, quizás con menor lucidez que en otras, qué ritmo de trabajo y de descanso merece la pena adoptar, cuántas horas dedicar al tiempo libre, a ver la televisión, a estar con los amigos... (Pastor, Balaguer y García-Merita, en prensa). También durante este periodo los hábitos relacionados con la alimentación y la actividad física pueden experimentar cambios importantes ejerciendo un considerable efecto los modelos estéticos imperantes que en la actualidad proponen la delgadez como prototipo de belleza (Vandereycken y Meerman, 1984).

Este trabajo trata de comprobar cómo perciben los adolescentes este tipo de demandas a veces contradictorias. La intervención educativa y psicosocial se ve facilitada cuando es posible disponer de una visión de conjunto de percepciones como las que aquí se plantean (el autoconcepto, los hábitos saludables, el bienestar personal) lo que permite identi- ficar diferentes flancos por donde poder incidir en el mantenimiento $\mathrm{u}$ optimización de los estilos de vida saludables y en la modificación o erradicación de aquellos otros que resultan perjudiciales para los individuos y para la comunidad.

\section{EL AUTOCONCEPTO FÍSICO}

Una variable muy relacionada con la adopción de conductas saludables 0 menos saludables es el autoconcepto. El autoconcepto siempre ha merecido una atención privilegiada por parte de la psicología como componente central del comportamiento humano pero, en su relación con la salud, los estudios iniciales se deben a Fitts (1972) y a Burns (1979). Los resultados obtenidos resultaron, en un principio, contradictorios debido en buena parte a que asumían una concepción unidimensional y global del autoconcepto que en nuestros dias no se considera adecuada. En efecto, desde mediados de los años setenta (Shavelson, Hubner y Stanton, 1976), se adopta de forma generalizada una concepción jerárquica y multidimensional del autoconcepto en la que se presta atención particularizada a dimensiones como la social, la académica, la personal o la física, que es la que ahora nos ocupa.

$\mathrm{El}$ autoconcepto físico experimenta un curso propio de desarrollo evolutivo internamente diferenciado en varios dominios (Goñi, Rodríguez y Ruiz de Azúa, 2004), guarda relación con los riesgos de padecer trastornos de conducta alimentaria (Goñi y Rodríguez, 2004) y muestra sensibilidad para captar diferencias asociadas a variables como el género, la edad, la frecuencia y el tipo de actividad deportiva o el índice de masa corporal (Goñi, Ruiz de Azúa y Rodríguez, 2004). Pero probablemente son muchas más las variables con las que 
guarda estrecha relación el autoconcepto físico entre las que cabe citar los estilos de vida saludable o el bienestar psicológico. Y esto es precisamente lo que se somete a revisión en este trabajo.

Aun cuando apenas existen estudios empíricos que la confirmen, es razonable la siguiente presunción que relaciona los estilos de vida con el autoconcepto físico: las personas están motivadas para actuar en las áreas en las que experimentan sentimientos positivos de competencia y estima, siendo también cierto lo contrario, es decir, que se muestra baja motivación hacia actividades propias de las áreas en las que la persona no se siente competente.

\section{ESTILOS DE VIDA SALUDABLES}

Por estilo de vida saludable se entiende el patrón de comportamientos relativamente estable de los individuos o grupos que guardan una estrecha relación con la salud (Nutbeam, Aaro y Catford, 1989). Un patrón conductual es el modo habitual de respuesta a diferentes situaciones; el consumo de cigarrillos y alcohol, las dietas ricas en grasas y abundantes o la falta de ejercicio constituyen algunos ejemplos de comportamientos poco saludables o de riesgo. Entre las variables más clásicas a considerar dentro de un estilo de vida saludable figuran la práctica deportiva, los hábitos de alimentación, el consumo del alcohol y de tabaco y también las percepciones de salud que experimentan los sujetos (Balaguer, 2002).

En un estudio que constituye un antecedente directo de esta investigación (Dolcini y Adler, 1994) no se encontró relación directa entre el consumo de sustancias tóxicas legales (tabaco y alcohol) y el autoconcepto físico. En cambio en el trabajo de Pastor et al. (en prensa), utili- zando el Perfil de Autopercepciones para Adolescentes (SPPA) de Harter, se encontraron correlaciones positivas de la percepción (autoconcepto) en competencia fisica con la práctica deportiva y con la alimentación sana, y negativas con el consumo de alcohol y tabaco; en cambio la dimensión de apariencia física ha mostrado relaciones menos claras con los hábitos de vida saludable. En todo caso, se trata de datos tan sugerentes como escasos y poco concluyentes por lo que nos encontramos ante una temática totalmente abierta a la investigación.

\section{BIENESTAR PSICOLÓGICO}

Desde mediados de los años setenta (Campbell, Converse y Rodgers, 1976; Andrews y Withey, 1976) el interés por la calidad de vida fomenta estudios sobre el modo y la intensidad con que las personas experimentan y valoran sus vidas de forma positiva (Diener, 1994; Veenhoven, 1994). Con ello se introducen en psicologia términos relativamente nuevos, como los de bienestar subjetivo o satisfacción con la vida, que sin embargo retoman tópicos centrales del comportamiento humano.

Tres factores básicos explican el bienestar psicológico (Bradburn, 1969; Diener, 1994; Huebner, 1991; Huebner y Dew, 1996): a) la satisfacción con la vida, entendida como la evaluación positiva de la vida en su conjunto; b) la frecuencia individual de emociones positivas o afecto positivo; c) la frecuencia individual de emociones negativas a la que se denomina afecto negativo. Ambos tipos de afecto correlacionan negativamente de tal modo que la presencia de uno anula la del otro. Cabe, por tanto, pensar que la vivencia subjetiva con la que el sujeto experiencia su vida guarda directa conexión con su autoconcepto, con la percepción/valoración de sí mismo. 


\section{OBJETIVOS}

Este trabajo tiene un marcado carácter prospectivo siendo su principal objetivo el de identificar las eventuales relaciones que puedan producirse entre las respuestas al CAF (Cuestionario de Autoconcepto Físico) y a la Escala de Bienestar Psicológico (EBP), y distintas variables (salud subjetiva, consumo de alcohol y de tabaco, actividad física...) que permiten definir un determinado estilo de vida de nuestros adolescentes.

La expectativa genérica en cuanto a resultados es que los indices más elevados en el CAF (autoconcepto físico más alto), así como el mayor grado de bienestar psicológico, correlacionen con los hábitos de vida más saludables. No se trata, por tanto, de estudiar los estilos de vida en la adolescencia (Balaguer, 2002) sino de identificar la relación que los estilos de vida guardan con rasgos psicológicos tan significativos como el autoconcepto físico y el bienestar psicológico.

\section{MATERIAL Y METODO}

\section{Muestra}

Participaron en este estudio un total de 539 estudiantes con edades comprendidas entre los 12 a 23 años; de ellos 181 estudiaban en tres centros de San Sebastián (un centro de Educación Secundaria y dos Facultades Universitarias) y 358 en cinco centros de VitoriaGasteiz (cuatro centros de Educación Secundaria y una Escuela Universitaria). La muestra dividida en función del género resultó equilibrada ya que se componía de 284 mujeres con una edad media de 17,03 años ( $\sigma=3,63)$ y de 256 hombres cuya edad media era de 15,66 años $(\sigma=3,63)$.

Para los análisis presentados en este estudio se considera a esta muestra como un solo grupo. Indudablemente los resultados que aquí se presentan admiten análisis complementarios tomando en consideración las eventuales diferencias asociadas tanto con el género como con la edad pero su exposición desbordaría las dimensiones de este artículo.

\section{Procedimiento}

La administración de los cuestionarios vino a exigir un tiempo aproximado de 45 minutos. Cabe destacar que a todos los encuestados se les aseguró el anonimato con el fin de poder reducir al máximo la probabilidad del efecto de deseabilidad social. Se imprimió, por otra parte, cada cuestionario en un color distinto para contribuir a hacer más agradable la tarea.

\section{Instrumentos}

El Cuestionario de Autoconcepto Físico (CAF) es un instrumento de medida (Goñi, Ruiz de Azúa y Liberal, 2004) que reúne unas aceptables propiedades psicométricas y que confirma la solidez del modelo hipotetizado en cuanto a la estructura interna (dimensiones) del autoconcepto físico. El cuestionario está formado por 36 ítems (6 ítems para cada escala), redactados en términos enunciativos, destinados a medir las siguientes dimensiones del autoconcepto físico:

1. Habilidad fisica. Percepción de las cualidades y habilidades para la práctica de los deportes; capacidad de aprender deportes; seguridad personal y predisposición ante los deportes. Ejemplo: "Practicando deportes soy una persona hábil”.

2. Condición física. Forma física; resistencia y energí; confianza en 
el estado físico. Ejemplo: "Puedo correr y hacer ejercicio durante mucho tiempo sin cansarme".

3. Atractivo físico. Percepción de la apariencia física propia: seguridad y satisfacción por la imagen propia. Ejemplo: EMe siento contento/a con mi imagen corporal".

4. Fuerza. Verse y/o sentirse fuerte, con capacidad para levantar peso, con seguridad ante ejercicios que exigen fuerza y con predisposición a realizar dichos ejercicios. Ejemplo: "Tengo más fuerza que la mayoría de la gente de mi edad".

5. Autoconcepto físico general. Opinión y sensaciones positivas (felicidad, satisfacción, orgullo y confianza) en lo físico. Ejemplo: "Físicamente me siento bien".

6. Autoconcepto general. Satisfacción con uno/a mismo/a y con la vida en general. Ejemplo: "No tengo demasiadas cualidades como persona”.

Como alternativa de respuesta a cada item se ofrecen cinco opciones en una escala tipo Likert donde $1=$ Falso, 2=Casi siempre falso, $3=\mathrm{A}$ veces verdadero, a veces falso, 4=Casi siempre verdadero, y $5=$ Verdadero, de tal modo que a mayor puntuación correspondería un mayor autoconcepto físico. Los coeficientes de fiabilidad (alfa de Cronbach) de cada una de sus escalas son los siguientes: Habilidad física $\alpha=0.8004$; Condición física $\alpha=0.8446$; Atractivo físico $\alpha=0.8778$; Fuerza $\alpha=0.8329$; Autoconcepto físico general $\mathrm{a}=0.8824$; $\mathrm{y}$ Autoconcepto general $\alpha=0.7887$. Los cuatro factores primeros del cuestionario explican un $60 \%$ de la varianza del test. Y, de otro lado, el CAF ha mostrado sensibilidad para discriminar entre las respuestas de personas de distintas edades así como entre hombres y mujeres.

Para evaluar el estilo de vida se ha redactado un Cuestionario sobre Hábitos de Vida (cf. anexo) a partir del Inventario de Conductas de Salud de Escolares (Wold, 1995). El inventario consta de 84 preguntas destinadas a evaluar el estilo de vida de los adolescentes, desde la frecuencia e intensidad de deporte practicado a la vida sexual, pasando por el consumo de tabaco, alcohol o drogas, el tipo de alimentación, la higiene bucal, la salud o el tiempo dedicado a ver la televisión; sus ítems están redactados en forma de pregunta con varias opciones de respuestas. De los 84 items que conforman el inventario se seleccionaron para este Cuestionario únicamente 5 con la finalidad de medir algunos de los hábitos de vida saludables, los más centrales (actividad fisica, consumo de alcohol y de tabaco, tipo de alimentación y nivel subjetivo de salud), sin pretender que las variables elegidas agoten todas las vertientes de lo que es una vida saludable. Lo que, por el momento, interesa comprobar es si, genéricamente, se producen correspondencias entre las puntuaciones en el CAF y la mayor o menor realización de conductas saludables. Puede decirse que este conjunto de conductas o de hábitos más o menos saludables vienen a configurar un estilo de vida.

Asímismo se administró la Escala de Bienestar Psicológico (EBP), la cual está destinada a evaluar el grado de bienestar psicológico que experimenta el individuo (Sánchez Cánovas, 1998). Para ello las respuestas se valoran en una escala Likert de cinco grados de modo que a mayor puntuación mayor indice de bienestar psicológico. El cuestionario está formado por 65 items que conforman 4 subescalas: Bienestar Psicológico Subjetivo; Bienestar material (mide la percepción subjetiva que tiene el sujeto de su situa- 
ción económica); Bienestar laboral (analiza la satisfacción con el trabajo o el empleo); y Relaciones con la Pareja. Los coeficientes de consistencia interna de cada una de las subescalas son los siguientes: Bienestar Psicológico Subjetivo $\alpha=0,9235$; Bienestar Material $\alpha=0,907$; Bienestar Laboral $\alpha=0,873$; Relaciones con la Pareja $\alpha=0,870$. En esta ocasión se aplicó una versión reducida del cuestionario EBP que comprendia 40 items correspondientes únicamente a las subescalas de Bienestar psicológico subjetivo (los 30 primeros items del cuestionario) y de Bienestar material (items del 31 al 40). Se seleccionaron únicamente esas dos escalas tras valorar que resultan significativas también para adolescentes menores de 17 años; no se aplicaron, en cambio, las otras dos escalas (Bienesta material y Relaciones con la pareja) porque no respondian a unos referentes comunes a toda la muestra de este estudio.

\section{Estadisticos}

Para analizar los datos se realizaron comparaciones de medias independien- tes, mediante la prueba T-test (tablas de la I a la IV), así como un análisis de la varianza (ANOVA) para la variable "Salud subjetiva" (tabla VI). En el caso de la variable "Alimentación" (tabla V) se empleó una prueba no paramétrica, concretamente la de Kruskal-Wallis, debido a que el tamaño de la muestra de uno de los subgrupos no superaba los 30 sujetos. La relación obtenida entre las repsuestas a los cuestionarios CAF y EBP (tabla VII) se determinó a través de la prueba de correlación de Pearson.

\section{RESULTADOS}

La tabla I divide a los partipantes en esta investigación en dos grandes grupos: el de quienes practican deporte $(\mathrm{N}=463 ; 86 \%)$ y el de quienes no lo practican $(\mathrm{N}=76 ; 14 \%)$, según respondieron a la primera pregunta sobre hábitos de vida (cf. Anexo).

Las puntuaciones en las distintas subescalas del CAF siempre son más altas en los sujetos que realizan actividad físico-deportiva, aun cuando en las

Tabla 1. Puntuaciones en el CAF y actividad física

\begin{tabular}{|c|c|c|c|c|c|c|}
\hline ESCALAS CAF & $\begin{array}{l}\text { ACTIVIDAD } \\
\text { FÍSICA }\end{array}$ & $\begin{array}{c}\mathrm{N} \\
540\end{array}$ & $\mathrm{X}$ & $\sigma$ & $\mathrm{t}$ & Sig. \\
\hline Habilidad Fisica & $\begin{array}{c}\text { No realiza } \\
\text { Realiza }\end{array}$ & $\begin{array}{c}77 \\
463\end{array}$ & $\begin{array}{l}17,92 \\
22,10\end{array}$ & $\begin{array}{l}5,686 \\
5,370\end{array}$ & $-6,226$ &, $000^{* * *}$ \\
\hline Atractivo Físico & $\begin{array}{c}\text { No realiza } \\
\text { Realiza }\end{array}$ & $\begin{array}{c}77 \\
463\end{array}$ & $\begin{array}{l}17,89 \\
20,39\end{array}$ & $\begin{array}{l}5,996 \\
5,969\end{array}$ & $-3,373$ &, $001 * * *$ \\
\hline Condición Física & $\begin{array}{c}\text { No realiza } \\
\text { Realiza }\end{array}$ & $\begin{array}{c}77 \\
463\end{array}$ & $\begin{array}{l}18,31 \\
20,11\end{array}$ & $\begin{array}{l}6,548 \\
5,936\end{array}$ & $-2,416$ & ,016* \\
\hline Fuerza & $\begin{array}{c}\text { No realiza } \\
\text { Realiza }\end{array}$ & $\begin{array}{c}77 \\
463\end{array}$ & $\begin{array}{l}15,72 \\
18,31\end{array}$ & $\begin{array}{l}3,684 \\
4,640\end{array}$ & $-5,457$ &, $000 * * *$ \\
\hline $\begin{array}{l}\text { Autoconcepto } \\
\text { Físico General }\end{array}$ & $\begin{array}{c}\text { No realiza } \\
\text { Realiza }\end{array}$ & $\begin{array}{c}77 \\
463\end{array}$ & $\begin{array}{l}21,02 \\
22,09\end{array}$ & $\begin{array}{l}7,006 \\
6,027\end{array}$ & $-1,262$ & ,210 \\
\hline $\begin{array}{l}\text { Autoconcepto } \\
\text { General }\end{array}$ & $\begin{array}{c}\text { No realiza } \\
\text { Realiza }\end{array}$ & $\begin{array}{c}77 \\
463\end{array}$ & $\begin{array}{l}23,17 \\
24,25\end{array}$ & $\begin{array}{l}4,934 \\
4,654\end{array}$ & $-1,864$ & ,063 \\
\hline
\end{tabular}


dos de orden superior, la de autoconcepto físico general y la de autoconcepto general, dichas diferencias no son significativas; estos datos se complementan con los de la tabla siguiente.

La tabla II confirma los resultados anteriores. Ahora la muestra queda dividida en el grupo de quienes practican deporte de forma esporádica $(\mathrm{N}=212$; $39 \%$ ) y el de quienes lo practican de forma regular $(\mathrm{N}=327 ; 61 \%)$. A tal fin se incluye en la práctica esporádica a quienes responden a la pregunta 1 del anexo que "no" hacen deporte o que lo practican "de vez en cuando", mientras que la forma regular supone practicar al menos de 1 a 3 veces por semana 0 incluso más de 3 veces.

Las diferencias son claras, y ahora significativas, en todas las subescalas del cuestionario en el sentido de que manifiestan un autoconcepto físico más elevado quienes practican deporte con regularidad.

La tabla III recoge los datos relativos al hábito de fumar. Las categorías de respuesta a la pregunta 2 sobre hábitos de vida se han reagrupado considerándose que "fuma" quien elige las dos primeras opciones (a diario; alguna vez a la semana, pero no todos los días) y que "no fuma" quien elige las restantes opciones.

Los no fumadores $(\mathrm{N}=307 ; 57 \%)$ ofrecen mejores indices que los fumadores $(\mathrm{N}=153 ; 43 \%)$ en todas las subescalas del CAF resultando significativas las diferencias en tres de éllas: habilidad fisica, condicion fisica y autoconcepto general; no alcanzan significatividad, sin embargo, en subescalas como las de atractivo fisico y fuerza. Encajan bien estos datos con el modelo teórico del autoconcepto físico.

La tabla IV refleja las diferencias en el autoconcepto de las personas catalogadas como bebedoras frente a las no bebedoras. Se incluye en la categoría de "bebe" a quien elige las dos primeras opciones de la pregunta 3 del cuestionario y en la de "no bebe" al resto, es decir, a quien bebe menos de una vez a la semana o no bebe nunca.

Tabla 2. Medida del autoconcepto físico y frecuencia de la práctica deportiva

\begin{tabular}{|c|c|c|c|c|c|c|}
\hline ESCALAS CAF & $\begin{array}{l}\text { ACTIVIDAD } \\
\text { FÍSICA }\end{array}$ & $\begin{array}{c}\mathrm{N} \\
540\end{array}$ & $\mathrm{X}$ & $\sigma$ & $\mathrm{t}$ & Sig. \\
\hline Habilidad Fisica & $\begin{array}{c}\text { Esporádica } \\
\text { Habitual }\end{array}$ & $\begin{array}{l}213 \\
327\end{array}$ & $\begin{array}{l}19,13 \\
23,05\end{array}$ & $\begin{array}{l}5,633 \\
5,017\end{array}$ & $-8,447$ &, $000^{* * *}$ \\
\hline Atractivo Físico & $\begin{array}{c}\text { Esporádica } \\
\text { Habitual }\end{array}$ & $\begin{array}{l}213 \\
327\end{array}$ & $\begin{array}{l}18,89 \\
20,77\end{array}$ & $\begin{array}{l}5,895 \\
6,010\end{array}$ & $-3,580$ &, $000 * * *$ \\
\hline Condición Fisica & $\begin{array}{c}\text { Esporádica } \\
\text { Habitual }\end{array}$ & $\begin{array}{l}213 \\
327\end{array}$ & $\begin{array}{l}18,12 \\
20,98\end{array}$ & $\begin{array}{l}6,341 \\
5,586\end{array}$ & $-5,492$ &, $000^{* * *}$ \\
\hline Fuerza & $\begin{array}{c}\text { Esporádica } \\
\text { Habitual }\end{array}$ & $\begin{array}{l}213 \\
327\end{array}$ & $\begin{array}{l}16,46 \\
18,91\end{array}$ & $\begin{array}{l}4,429 \\
4,462\end{array}$ & $-6,247$ &, $000 * * *$ \\
\hline $\begin{array}{l}\text { Autoconcepto } \\
\text { Fisico General }\end{array}$ & $\begin{array}{c}\text { Esporádica } \\
\text { Habitual }\end{array}$ & $\begin{array}{l}213 \\
327\end{array}$ & $\begin{array}{l}20,99 \\
22,56\end{array}$ & $\begin{array}{l}6,468 \\
5,912\end{array}$ & $-2,910$ &, $004^{* *}$ \\
\hline $\begin{array}{c}\text { Autoconcepto } \\
\text { General }\end{array}$ & $\begin{array}{c}\text { Esporádica } \\
\text { Habitual }\end{array}$ & $\begin{array}{l}213 \\
327\end{array}$ & $\begin{array}{l}23,47 \\
24,50\end{array}$ & $\begin{array}{l}4,858 \\
4,565\end{array}$ & $-2,496$ & ,013* \\
\hline
\end{tabular}


Tabla 3. Autoconcepto físico y consumo de tabaco

\begin{tabular}{|c|c|c|c|c|c|c|}
\hline ESCALAS CAF & $\begin{array}{l}\text { CONSUMO } \\
\text { DE TABACO }\end{array}$ & $\begin{array}{c}\mathrm{N} \\
540\end{array}$ & $X$ & $\sigma$ & $\mathrm{t}$ & Sig. \\
\hline Habilidad Fisica & $\begin{array}{c}\text { Fuma } \\
\text { No fuma }\end{array}$ & $\begin{array}{l}153 \\
387\end{array}$ & $\begin{array}{l}20,48 \\
21,93\end{array}$ & $\begin{array}{l}5,680 \\
5,521\end{array}$ & 2,731 &, $007 * *$ \\
\hline Atractivo Fisico & $\begin{array}{c}\text { Fuma } \\
\text { No fuma }\end{array}$ & $\begin{array}{l}153 \\
387\end{array}$ & $\begin{array}{l}19,38 \\
20,30\end{array}$ & $\begin{array}{l}5,575 \\
6,183\end{array}$ & 1,606 &, 109 \\
\hline Condición Fisica & $\begin{array}{c}\text { Fuma } \\
\text { No fuma }\end{array}$ & $\begin{array}{l}153 \\
387\end{array}$ & $\begin{array}{l}18,99 \\
20,21\end{array}$ & $\begin{array}{l}6,320 \\
5,911\end{array}$ & 2,117 & ,035* \\
\hline Fuerza & $\begin{array}{c}\text { Fuma } \\
\text { No fuma }\end{array}$ & $\begin{array}{l}153 \\
387\end{array}$ & $\begin{array}{l}17,73 \\
18,05\end{array}$ & $\begin{array}{l}4,762 \\
4,551\end{array}$ &, 729 & , 466 \\
\hline $\begin{array}{l}\text { Autoconcepto } \\
\text { Físico General }\end{array}$ & $\begin{array}{c}\text { Fuma } \\
\text { No fuma }\end{array}$ & $\begin{array}{l}153 \\
387\end{array}$ & $\begin{array}{l}21,33 \\
22,19\end{array}$ & $\begin{array}{l}6,011 \\
6,232\end{array}$ & 1,461 &, 145 \\
\hline $\begin{array}{c}\text { Autoconcepto } \\
\text { General }\end{array}$ & $\begin{array}{c}\text { Fuma } \\
\text { No fuma }\end{array}$ & $\begin{array}{l}153 \\
387\end{array}$ & $\begin{array}{l}22,98 \\
24,52\end{array}$ & $\begin{array}{l}4,427 \\
4,749\end{array}$ & 3,463 &, $001 * * *$ \\
\hline
\end{tabular}

Tabla 4. Autoconcepto físico y consumo de alcohol

\begin{tabular}{|c|c|c|c|c|c|c|}
\hline ESCALAS CAF & $\begin{array}{c}\text { CONSUMO } \\
\text { DE ALCOHOL }\end{array}$ & $\begin{array}{c}\mathrm{N} \\
540\end{array}$ & Media & $\sigma$ & $\mathrm{t}$ & Sig. \\
\hline Habilidad Fisica & $\begin{array}{c}\text { Bebe } \\
\text { No bebe }\end{array}$ & $\begin{array}{l}249 \\
291\end{array}$ & $\begin{array}{l}20,83 \\
22,11\end{array}$ & $\begin{array}{l}5,538 \\
5,603\end{array}$ & $-2,654$ &, $008^{* *}$ \\
\hline Atractivo Físico & $\begin{array}{c}\text { Bebe } \\
\text { No bebe }\end{array}$ & $\begin{array}{l}249 \\
291\end{array}$ & $\begin{array}{l}19,67 \\
20,36\end{array}$ & $\begin{array}{l}5,607 \\
6,362\end{array}$ & $-1,351$ &, 177 \\
\hline Condición Física & $\begin{array}{c}\text { Bebe } \\
\text { No bebe }\end{array}$ & $\begin{array}{l}249 \\
291\end{array}$ & $\begin{array}{l}19,03 \\
20,59\end{array}$ & $\begin{array}{l}6,000 \\
6,009\end{array}$ & $-3,017$ &, $003^{* *}$ \\
\hline Fuerza & $\begin{array}{c}\text { Bebe } \\
\text { No bebe }\end{array}$ & $\begin{array}{l}249 \\
291\end{array}$ & $\begin{array}{l}17,67 \\
18,21\end{array}$ & $\begin{array}{l}4,818 \\
4,421\end{array}$ & $-1,343$ &, 180 \\
\hline $\begin{array}{l}\text { Autoconcepto } \\
\text { Fisico General }\end{array}$ & $\begin{array}{c}\text { Bebe } \\
\text { No bebe }\end{array}$ & $\begin{array}{l}249 \\
291\end{array}$ & $\begin{array}{l}21,26 \\
22,54\end{array}$ & $\begin{array}{l}5,967 \\
6,310\end{array}$ & $-2,413$ & ,016* \\
\hline $\begin{array}{c}\text { Autoconcepto } \\
\text { General }\end{array}$ & $\begin{array}{c}\text { Bebe } \\
\text { No bebe }\end{array}$ & $\begin{array}{l}249 \\
291\end{array}$ & $\begin{array}{l}23,02 \\
25,01\end{array}$ & $\begin{array}{l}4,722 \\
4,513\end{array}$ & $-4,994$ &, $000 * * *$ \\
\hline
\end{tabular}

De nuevo, las diferencias en autoconcepto favorecen al grupo que "no bebe" $(\mathrm{N}=291 ; 54 \%)$ frente al grupo que "bebe" $(\mathrm{N}=248 ; 46 \%)$. Las dimensiones menos asociadas con los hábitos de consumo de bebidas alcohólicas son la de fuerza y la de atractivo (como sucedía en relación con el tabaco) mientras que en el resto se trata de diferencias estadísticamente significativas.

En la tabla $V$ se cruzan los datos de respuesta al CAF con la percepción de la propia alimentación para la que la pregunta 4 del cuestionario ofrecía cinco opciones que se han agrupado en tres: 
Autoconcepto fisico y estilos de vida en la adolescencia

Tabla 5. Puntuaciones en el CAF y percepción de la alimentación propia

\begin{tabular}{|c|c|c|c|c|c|c|}
\hline ESCALAS CAF & ALIMENTACIÓN & $\begin{array}{c}\mathrm{N} \\
540\end{array}$ & $\mathrm{X}$ & $\sigma$ & $\chi^{2}$ & Sig. \\
\hline Habilidad Fisica & $\begin{array}{c}\text { Menos sana } \\
\text { Igual de sana } \\
\text { Más sana }\end{array}$ & $\begin{array}{c}15 \\
434 \\
91\end{array}$ & $\begin{array}{l}18,64 \\
21,34 \\
22,84\end{array}$ & $\begin{array}{l}6,481 \\
5,404 \\
6,123\end{array}$ & 10,078 &, $006^{* *}$ \\
\hline Atractivo Fisico & $\begin{array}{c}\text { Menos sana } \\
\text { Igual de sana } \\
\text { Más sana }\end{array}$ & $\begin{array}{c}15 \\
434 \\
91\end{array}$ & $\begin{array}{l}16,63 \\
19,99 \\
20,84\end{array}$ & $\begin{array}{l}7,215 \\
5,912 \\
6,214\end{array}$ & 5,491 &, 064 \\
\hline Condición Física & $\begin{array}{c}\text { Menos sana } \\
\text { Igual de sana } \\
\text { Más sana }\end{array}$ & $\begin{array}{c}15 \\
434 \\
91\end{array}$ & $\begin{array}{l}15,53 \\
19,58 \\
21,91\end{array}$ & $\begin{array}{l}7,180 \\
5,954 \\
5,731\end{array}$ & 17,507 &, $000 * * *$ \\
\hline Fuerza & $\begin{array}{c}\text { Menos sana } \\
\text { Igual de sana } \\
\text { Más sana }\end{array}$ & $\begin{array}{c}15 \\
434 \\
91\end{array}$ & $\begin{array}{l}16,92 \\
17,83 \\
18,73\end{array}$ & $\begin{array}{l}5,031 \\
4,553 \\
4,768\end{array}$ & 3,623 &, 163 \\
\hline $\begin{array}{l}\text { Autoconcepto } \\
\text { Fisico General }\end{array}$ & $\begin{array}{c}\text { Menos sana } \\
\text { Igual de sana } \\
\text { Más sana }\end{array}$ & $\begin{array}{c}15 \\
434 \\
91\end{array}$ & $\begin{array}{l}15,91 \\
21,82 \\
23,58\end{array}$ & $\begin{array}{l}7,893 \\
5,972 \\
6,193\end{array}$ & 18,743 &, $000^{* * *}$ \\
\hline $\begin{array}{c}\text { Autoconcepto } \\
\text { General }\end{array}$ & $\begin{array}{l}\text { Menos sana } \\
\text { Igual de sana } \\
\text { Más sana }\end{array}$ & $\begin{array}{c}15 \\
434 \\
91\end{array}$ & $\begin{array}{l}19,79 \\
23,98 \\
25,30\end{array}$ & $\begin{array}{l}6,707 \\
4,613 \\
4,331\end{array}$ & 13,869 &, $001^{* * *}$ \\
\hline
\end{tabular}

grupo que considera que su alimentación es menos sana (opciones de "algo menos sana" y "bastante menos sana") y que lo conforman 15 personas (un 3\%); grupo mayoritario $(\mathrm{N}=434 ; 80 \%)$ que cree alimentarse de forma igual de sana que la gente de su edad; y el grupo $(\mathrm{N}=91 ; 17 \%)$ que cree alimentarse mejor ("más sano" o "un poco más sano").

Estos datos marcan una dirección clara ya que, en todos los casos, el autoconcepto decrece del grupo que mejor cree alimentarse al que peor quedando en una situación intermedia quienes creen que su alimentación viene a ser igual de sana que la de los demás. Las diferencias son estadísticamente significativas en todos los casos salvo en la dimensión fuerza del autoconcepto físico; las diferencias más importantes se si- túan, de todos modos, entre el pequeño grupo (3\%) de los que se alimentan peor y el resto.

Las agrupaciones en cuanto a salud subjetiva (Tabla VI) reflejaron diferencias significativas en todas las subescalas del $\mathrm{CAF}$.

El grupo de salud subjetiva "muy buena" $(\mathrm{N}=115 ; 21 \%)$ ofrece indices superiores a los del grupo de salud "bastante buena" ( $\mathrm{N}=309 ; 57 \%)$ y éste a su vez indices más altos en autoconcepto físico que el grupo de salud "nada buena" ( $\mathrm{N}=116 ; 22 \%)$, categoría que incluye tanto a quienes se creen "algo sanos" como a los que se ven "nada sanos". Los resultados se sitúan, en definitiva, en la misma dirección que los de las tablas anteriores. 
Tabla 6. Nivel subjetivo de salud y autoconcepto fisico

\begin{tabular}{|c|c|c|c|c|c|c|}
\hline ESCALAS CAF & $\begin{array}{c}\text { SALUD } \\
\text { SUBJETIVA }\end{array}$ & $\stackrel{\mathrm{N}}{540}$ & $\mathrm{X}$ & $\sigma$ & $\mathrm{F}$ & Sig. \\
\hline Habilidad Física & $\begin{array}{l}\text { Nada buena } \\
\text { Bastante buena } \\
\text { Muy buena }\end{array}$ & $\begin{array}{l}115 \\
309 \\
116\end{array}$ & $\begin{array}{l}19,77 \\
21,45 \\
23,43\end{array}$ & $\begin{array}{l}5,627 \\
5,338 \\
5,704\end{array}$ & 12,884 &, $000 * * *$ \\
\hline Atractivo Físico & $\begin{array}{c}\text { Nada buena } \\
\text { Bastante buena } \\
\text { Muy buena }\end{array}$ & $\begin{array}{l}115 \\
309 \\
116\end{array}$ & $\begin{array}{l}17,72 \\
20,17 \\
22,00\end{array}$ & $\begin{array}{l}6,322 \\
5,710 \\
5,826\end{array}$ & 15,562 &, $000 * * *$ \\
\hline Condición Física & $\begin{array}{c}\text { Nada buena } \\
\text { Bastante buena } \\
\text { Muy buena }\end{array}$ & $\begin{array}{l}115 \\
309 \\
116\end{array}$ & $\begin{array}{l}17,23 \\
19,76 \\
22,75\end{array}$ & $\begin{array}{l}6,381 \\
5,694 \\
5,381\end{array}$ & 26,419 &, $000 * * *$ \\
\hline Fuerza & $\begin{array}{c}\text { Nada buena } \\
\text { Bastante buena } \\
\text { Muy buena }\end{array}$ & $\begin{array}{l}115 \\
309 \\
116\end{array}$ & $\begin{array}{l}17,34 \\
17,81 \\
18,96\end{array}$ & $\begin{array}{l}4,548 \\
4,498 \\
4,842\end{array}$ & 3,984 & ,019* \\
\hline $\begin{array}{l}\text { Autoconcepto } \\
\text { Fisico General }\end{array}$ & $\begin{array}{c}\text { Nada buena } \\
\text { Bastante buena } \\
\text { Muy buena }\end{array}$ & $\begin{array}{l}115 \\
309 \\
116 \\
\end{array}$ & $\begin{array}{l}18,91 \\
22,00 \\
24,83 \\
\end{array}$ & $\begin{array}{l}6,736 \\
5,718 \\
5,354 \\
\end{array}$ & 29,339 &, $000 * * *$ \\
\hline $\begin{array}{c}\text { Autoconcepto } \\
\text { General }\end{array}$ & $\begin{array}{c}\text { Nada buena } \\
\text { Bastante buena } \\
\text { Muy buena }\end{array}$ & $\begin{array}{l}115 \\
309 \\
116\end{array}$ & $\begin{array}{l}21,37 \\
24,51 \\
25,65\end{array}$ & $\begin{array}{l}5,282 \\
4,121 \\
4,521\end{array}$ & 29,583 &, $000 * * *$ \\
\hline
\end{tabular}

${ }^{*} \mathrm{p}<0,05 ;{ }^{* *} \mathrm{p}<0,01 ;{ }^{* * *} \mathrm{p}<0,001$

La tabla VII ofrece correlaciones entre el CAF y el EBP.

Tabla 7. Correlaciones entre CAF y EBP

\begin{tabular}{|c|c|c|c|c|}
\hline & & & \multicolumn{2}{|c|}{ EBP } \\
\hline & & & $\begin{array}{c}\text { Bienestar } \\
\text { Psicológico Subjetivo }\end{array}$ & Bienestar Material \\
\hline \multirow{6}{*}{ CAF } & Habilidad Fisica & $\begin{array}{c}\text { R de Pearson } \\
\text { Sig. }\end{array}$ & $\begin{array}{l}, \mathbf{3 7 0}(* * *) \\
, 000\end{array}$ & $\begin{array}{l}, \mathbf{3 4 8}(* * *) \\
, 000\end{array}$ \\
\hline & Atractivo Físico & $\begin{array}{c}\text { R de Pearson } \\
\text { Sig. }\end{array}$ & $\begin{array}{c}\mathbf{, 4 4 5}(* * *) \\
, 000\end{array}$ & $\begin{array}{c}\text {,348(***) } \\
, 000\end{array}$ \\
\hline & Condición Física & $\begin{array}{l}\text { R de Pearson } \\
\text { Sig. }\end{array}$ & $\begin{array}{c}. \mathbf{5 6 8}(* * *) \\
, 000\end{array}$ & $\begin{array}{c}\mathbf{4 5 3}(* * *) \\
, 000\end{array}$ \\
\hline & Fuerza & $\begin{array}{c}\text { R de Pearson } \\
\text { Sig. }\end{array}$ & $\begin{array}{c}\text {,388(***) } \\
\text {,000 }\end{array}$ & $\begin{array}{c}\text {,342(***) } \\
, 000\end{array}$ \\
\hline & $\begin{array}{l}\text { Autoconcepto } \\
\text { Fisico General }\end{array}$ & $\begin{array}{l}\text { R de Pearson } \\
\text { Sig. }\end{array}$ & $\begin{array}{c}\mathbf{5 8 4}(* * *) \\
, 000\end{array}$ & $\begin{array}{c}\mathbf{4 7 9}(* * *) \\
, 000 \\
\end{array}$ \\
\hline & $\begin{array}{l}\text { Autoconcepto } \\
\text { General }\end{array}$ & $\begin{array}{c}\text { R de Pearson } \\
\text { Sig. }\end{array}$ & $\begin{array}{c}\mathbf{6 0 9}^{* * * *)} \\
, 000\end{array}$ & $\begin{array}{c}, \mathbf{5 5 1}(* * *) \\
, 000\end{array}$ \\
\hline
\end{tabular}


En todos los casos los índices de correlación son altamente significativos $(\mathrm{p}<0,001)$ entre todas y cada una de las subescalas del CAF y de las subescalas del EBP. Quiere esto decir que se correponden las puntuaciones altas y bajas en el autoconcepto físico, medido mediante el CAF, con las puntuaciones altas y bajas tanto en bienestar psicológico subjetivo como en bienestar psicológico material, tal como mide estas dimensiones la Escala de Bienestar Personal, de Sánchez Cánovas.

\section{DISCUSION}

Son muchas y variadas las razones para llevar un estilo de vida saludable a la vez que son muy fuertes los factores socioculturales que juegan en contra de los buenos propósitos de hacer más ejercicio, de reducir la ingesta de alcohol o de alimentos, de llevar en definitiva una vida más sana. Por eso mismo es relevante identificar qué mecanismos psicológicos se asocian con el mantenimiento de un estilo saludable de vida. Y uno de esos mecanismos es, tal como lo ponen de relieve los datos presentados en este artículo, el autoconcepto físico: una vida poco saludable conlleva una percepción negativa mientras que el autoconcepto positivo va de la mano de los hábitos sanos.

Pero, una vez identificada la correlación, lo que falta por esclarecer es cuál es el sentido de la causalidad entre ambos factores: ¿vivir de forma sana incide en la mejora del autoconcepto físico? o, por el contrario, ¿un buen autoconcepto físico resulta un factor motivante para adoptar hábitos saludables? Tan verosimil es que un autoconcepto bajo en la condición fisica propia provenga de la poca actividad que el sujeto despliega como que quien se siente físicamente incompetente tiende a rehuir acti- vidades fisicas saludables. Posiblemente un estido vida poco saludable (sedentarismo, consumo de alcohol y/o tabaco, alimentación inadecuada...) derive de un autoconcepto físico negativo pero pudiera suceder que sea la conducta poco saludable la que sostenga el autoconcepto bajo. No se conoce, en este momento, con precisión cuál es la dirección de la causalidad aunque es muy probable que los onflujos se produzcan con carácter bidireccional.

En todo caso, tiene importancia el haber dejado constancia de la relación entre el autoconcepto, el bienestar psicológico y la vida saludable así como la tiene el proseguir la búsqueda de conexiones entre éstas y otras variables tanto individuales (personalidad, ideas, valores, modos de entender la vida...) como contextuales (familia, grupo de amigos...). Y es que, a diferencia de quienes estudian procesos psicológicos o sociológicos básicos, a una perspectiva de intervención educativa y psicosocial más que los procesos aislados lo que le interesa es la interrelación de los mimos. Valgan como muestra las siguientes consideraciones sobre los factores cuya interrelación ha quedado empíricamente constatada.

Los programas de intervención deberían poner de relieve y ayudar a tomar conciencia de que un esfuerza personal por mejorar el estilo de vida va a resultar rentable pero no sólo por sus repercusiones positivas en la salud física sino también en la salud psicológica: va a aumentar el bienestar psicológico, va a mejorar el autoconcepto. De otro lado, es decisivo, cuando se trata de un programa de intervención para la mejora de un determinado proceso (por ejemplo, los estilos de vida), el abordarlo tanto de forma directa como de manera indirecta, es decir, incidiendo en los factores asociados (en este caso, el autoconcepto y el bienestar psico- 
lógico) ya que previsiblemente la mejora de estos repercutirá en la mejora de los hábitos de vida saludables.

Por último, parece oportuno subrayar la necesidad de disponer de instrumentos que permitan detectar y diagnosticar de la forma más eficiente, económica y ajustada los casos que demandan una atención preventiva. Los contextos escolares, por ejemplo, son especialmente adecuados para llevar a cabo esta importante tarea de detección precoz y de intervención educativa y en estos contextos resulta útil y fácil contar con medidas del autoconcepto físico del alumna- do: el CAF se ha mostrado como un instrumento con sensibilidad para captar diferencias asociadas no sólo con las varibles estudiadas en este artículo (bienestar y estilos de vida) sino con otras variables tan significativas como los trastornos de conducta alimentaria y el indice de masa corporal (Goñi y Rodríguez, 2004). Así mismo ha podido comprobarse (Ruiz de Azúa, Rodríguez y Goñi, en prensa) que una mayor vulnerabilidad ante las presiones socioculturales en cuanto a la difusión del modelo estético de la delgadez corporal se asocia también con un autoconcepto físico menor.

\section{ANEXO \\ CUESTIONARIO SOBRE HÁBITOS DE VIDA}

1. En tu tiempo libre ¿realizas algún tipo de actividad física o deportiva?:

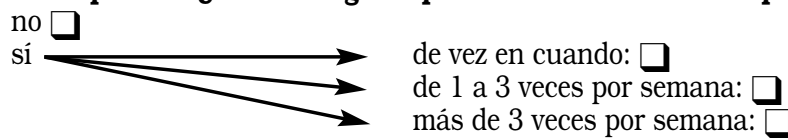

2. ¿Con cuánta frecuencia fumas, actualmente?

a diario:

alguna vez a la semana, pero no todos los días:

menos de una vez a la semana:

no fumo: ù

he dejado de fumar (por lo menos hace un mes):

3. ¿Con qué frecuencia consumes bebidas alcohólicas, aunque sólo sea en pequeña cantidad?

a diario:

alguna vez a la semana, pero no todos los días:

menos de una vez a la semana:

no bebo:

he dejado de beber alcohol por completo (por lo menos hace un mes):

4. ¿Cómo crees que es tu tipo de alimentación en comparación con la gente de tu edad?

más sano: $\square$

un poco más sano: $\square$

igual de sano: $\square$

algo menos sano:

bastante menos sano: $\square$

5. ¿Cómo crees que estás de sano/a?

muy sano/a:

bastante sano/a:

algo sano/a:

nada sano/a: 


\section{BIBLIOGRAFÍA}

Andrews, F., y Withey, S. B. (1976). Social indicators of well-being. New York: Plenum.

Balaguer, I. (Ed.) (2002). Estilos de vida en la adolescencia. Valencia: Promolibro.

Bradburn, N. M. (1969). The structure of psychological well-being. Chicago: Aldine.

Burns, R. B. (1979). The self-concept. Theory, measurement, development and behavior. New York: Longman.

Campbell, A., Converge, P. E., y Rodgers, W. L. (1976). The quality of american life. New York: Russell Sage.

Diener, E. (1994). El bienestar subjetivo. Intervención Psicosocial, 3(8), 67-113.

Dolcini, M. M., y Adler, N. E. (1994). Perceived competences, peer group affiliation, and risk behavior among adolescents. Health Psychology, 13(6), 496-506.

Fitts, W. H. (1972). The self-concept and behavior: Overview and supplement. Tennesee: Nashville.

Goñi, A. (2000). Psicología del individualismo. San Sebastián: Erein.

Goñi, A., Rodríguez, A., y Ruiz de Azúa, S. (2004). Bienestar psicológico y autoconcepto físico en la adolescencia y juventud. Psiquis, 25 (4), 141151

Goñi, A., Ruiz de Azúa, S., y Liberal, I. (2004). Las propiedades psicométricas de un nuevo cuestionario para la medida del autoconcepto físico. Revista de Psicología del Deporte, 13 (2), 195-213.

Goñi, A., Ruiz de Azúa, S., y Rodríguez, A. (2004) Deporte y autoconcepto físico en la preadolescencia. APUNTS. Educación Física y Deportes, $77,18-24$

Goñi, A., y Rodríguez, A. (2004). Trastornos de la conducta alimentaria, práctica deportiva y autoconcepto físico en adolescentes. Actas Españolas de Psiquiatría, 32(1), 29-36.

Huebner, E. S. (1991). Further validation of the Students' Life Satisfaction Scale: The independence of satisfaction and affect rating. Journal of Psychoeducational Assessment, 9, 363-368.

Huebner, E. S., y Dew, T. (1996). Interrelationships of positive affect, negative affect and life satisfaction in an adolescent sample. Social Indicators Research, 38, 129-137.

Nutbeam, D., Aaro, L., y Carford, J. (1989). Understanding children's health behavior: The impication for health promotion for young people. Social Science \& Medicine, 29(3), 317-325.

Pastor, Y., Balaguer, I., y García-Merita, M. L. (en prensa). Estilo de vida saludable en la adolescencia media: Análisis diferencial por curso y sexo. Revista de Psicología de la Salud.

Ruiz de Azúa, S., Rodríguez, A., y Goñi, A (2005). Variables socioculturales en la construcción del autoconcepto físico. Cultura y Educación, 17(3)

Sánchez-Cánovas, J. (1998). Escala de Bienestar Psicológico. Manual. Madrid: TEA.

Shavelson, R. J., Hubner, J. J., y Stanton, J. C. (1976). Self concept: Validation of construct interpretations. Review of Educational Research, 46, 407-441.

Vandereycken, W., y Meerman, R. (1984). Anorexia nervosa: Is prevention possible? International Journal of Psychiatry in Medicine, 3, 15-24.

Veenhoven, R. (1994). El estudio de la satisfacción con la vida. Intervención Psicosocial, 3(9), 87 116.

Wold, B. (1995). Health-behavior in schoolchildren: A who cross-national survey. Norway: University of Bergen. 